\title{
Lipoma across the Wall of the Right Atrium
}

\author{
WEI FAN ${ }^{1}$, Bin $\mathrm{Liao}^{2}$, and Xin $\mathrm{Li}^{2}$ \\ ${ }^{1}$ Affiliation not available \\ ${ }^{2}$ The Affiliated Hospital of Southwest Medical University
}

May 26, 2021

\begin{abstract}
A 44-year-old male patient was referred to our department with unremarkable physical examination and laboratory data due to a mass which was incidentally found in the right atrial during a routine examination. Transthoracic and transesophageal echocardiography revealed a $46 \times 30 \mathrm{~mm}$, well-delimited, non-mobile mass in the superior portion of the right atrium. Besides the intracardiac mass, another low density was detected in adjacent pericardial cavity at cardiac computed tomography ;he extracardiac mass appeared to be caused by invasive growth from the intracardiac mass. An operation was performed through right anterolateral minithoracotomy with the patient under hypothermic cardiopulmonary bypass. During operation, it was found that the surface of the right atrium was covered by an adipose mass $(30 \times 40 \mathrm{~mm}$; Fig. $2 \mathrm{~A})$. Intracardiac mass also showed yellow adipose tissue $(40 \times 50 \mathrm{~mm}$; Fig. $2 \mathrm{~B})$. Both parts of the mass infiltrated the myocardium. The mass was resected completely; and right atrium was reconstructed by using bovine pericardium pad. After the operation, the pathology confirmed the both intracardiac and extracardiac tissues as lipoma; transthoracic echocardiogram showed the atrial mass was removed completely and the left ventricular ejection fraction was normal . The patient's postoperative course was uneventful and he was discharged home after 7 days.
\end{abstract}

Lipoma across the Wall of the Right Atrium

Wei Fan MMS | Bin Liao MD | Xin Li MMS

Department of Cardiovascular Surgery, Affiliated Hospital of Southwest Medical University, Luzhou, China Correspondence

Bin Liao, MD, Department of Cardiovascular Surgery, Affiliated Hospital of Southwest Medical University, 25 Taiping Street, Jiangyang District, Luzhou, Sichuan Province 646000, China.

Email: fanwei1232021@163.com

\section{Funding}

No funding.

\section{Ethics approval and consent to participate}

All data collected in our study were obtained all necessary administrative permissions to access and subsequently use.

\section{Consent for publication}

Not applicable.

\section{Competing interests}

The authors declare that they have no competing interests. 


\section{Informed consent}

The patient has signed consent form, agreed to participate in this study, and allowed the researcher to publish photos of all cases without any restrictions on use.

This study does not apply to the guidelines for clinical trials.

The data that support the findings of this study are available on request from the corresponding author. The data are not publicly available due to privacy or ethical restrictions.

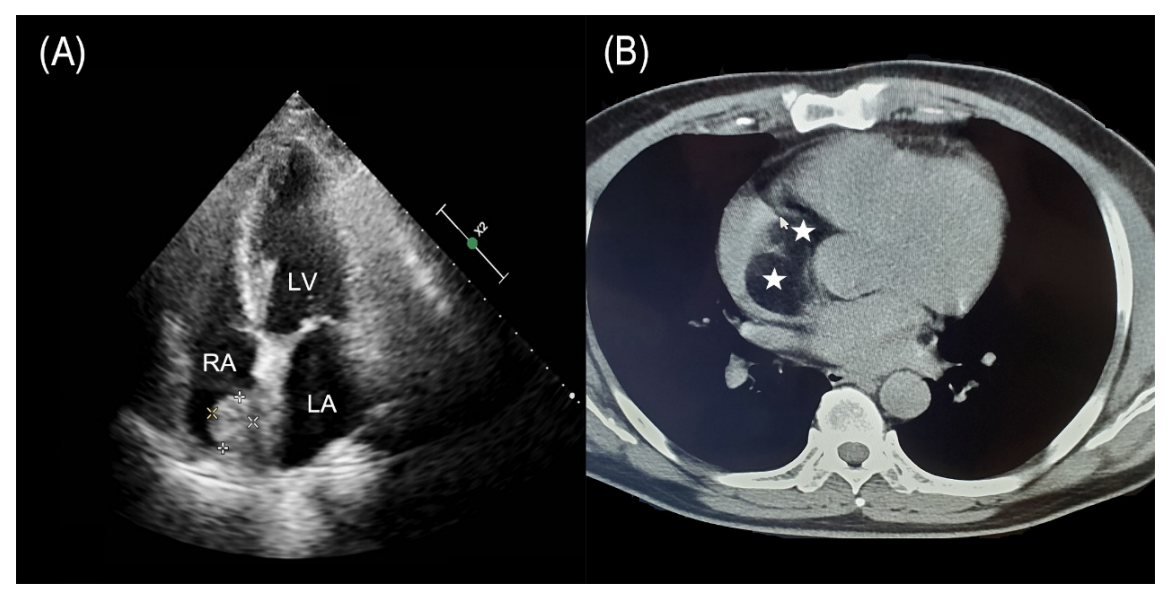

A 44-year-old male patient was referred to our department with unremarkable physical examination and laboratory data due to a mass which was incidentally found in the right atrial during a routine examination. His electrocardiogram displayed a normal sinus rhythm without related symptoms. Transthoracic and transesophageal echocardiography revealed a $46 \times 30 \mathrm{~mm}$, well-delimited, non-mobile mass (asterisks) in the superior portion of the right atrium (Fig.1A ). Besides the intracardiac mass, another low density was detected in adjacent pericardial cavity at cardiac computed tomography (Fig. $1 \boldsymbol{B}$ ); the extracardiac mass appeared to be caused by invasive growth from the intracardiac mass. An operation was performed through right anterolateral minithoracotomy with the patient under hypothermic cardiopulmonary bypass. During operation, it was found that the surface of the right atrium was covered by an adipose mass $(30 \times 40 \mathrm{~mm}$; Fig. $2 \boldsymbol{A})$. Intracardiac mass also showed yellow adipose tissue $(40 \times 50 \mathrm{~mm}$; Fig. $2 \boldsymbol{B})$. Both parts of the mass infiltrated the myocardium. The mass was resected completely; and right atrium was reconstructed by using bovine pericardium pad. After the operation, the pathology confirmed the both intracardiac and extracardiac tissues as lipoma (Fig.3 ); transthoracic echocardiogram showed the atrial mass was removed completely and the left ventricular ejection fraction was normal (Fig.4 ). The patient's postoperative course was uneventful and he was discharged home after 7 days. 

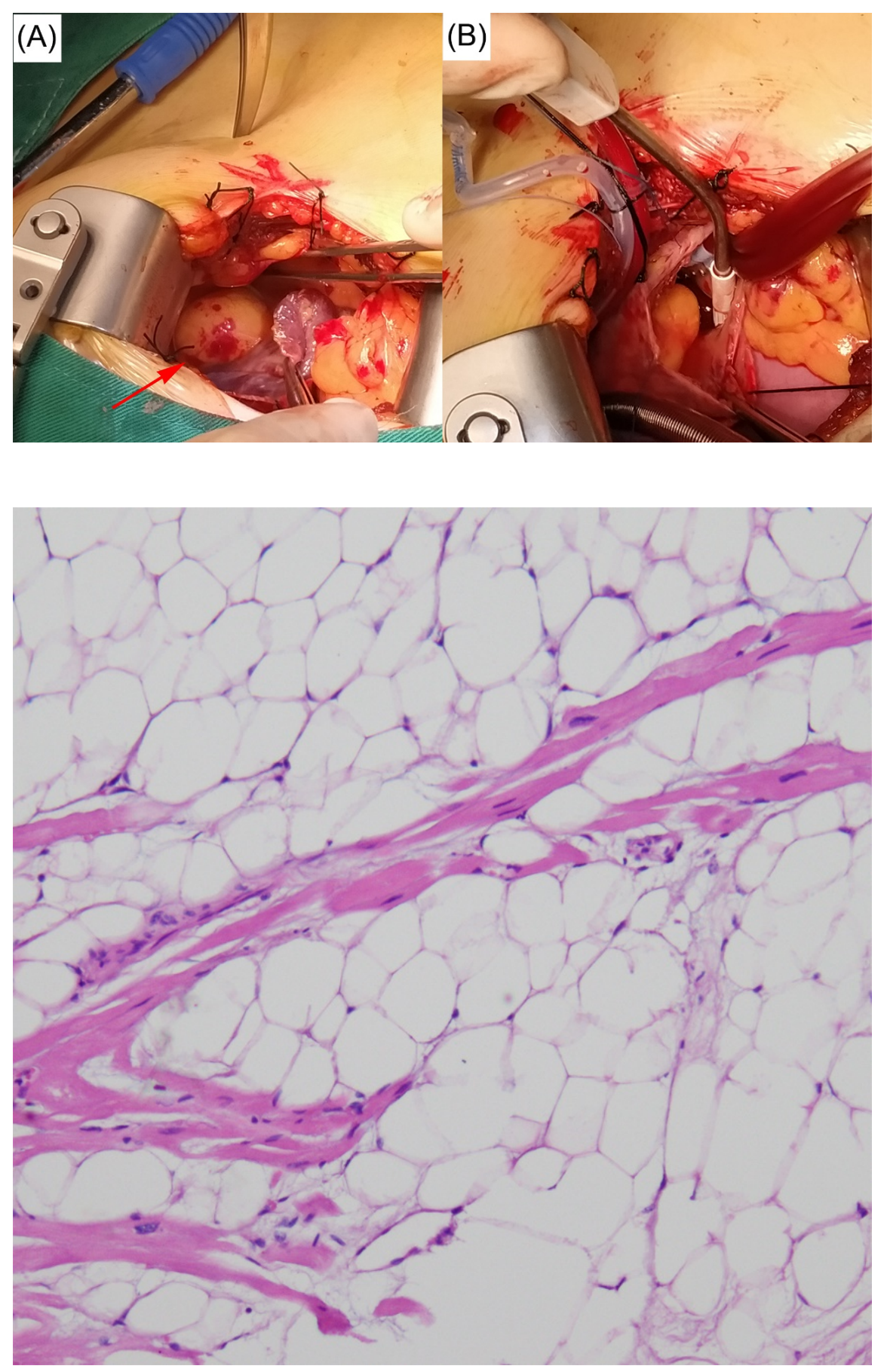

Figure 3 : Histology revealed both parts of the mass was lipoma with large number of adipose cells and little fibrous tissue (H\&E stain 


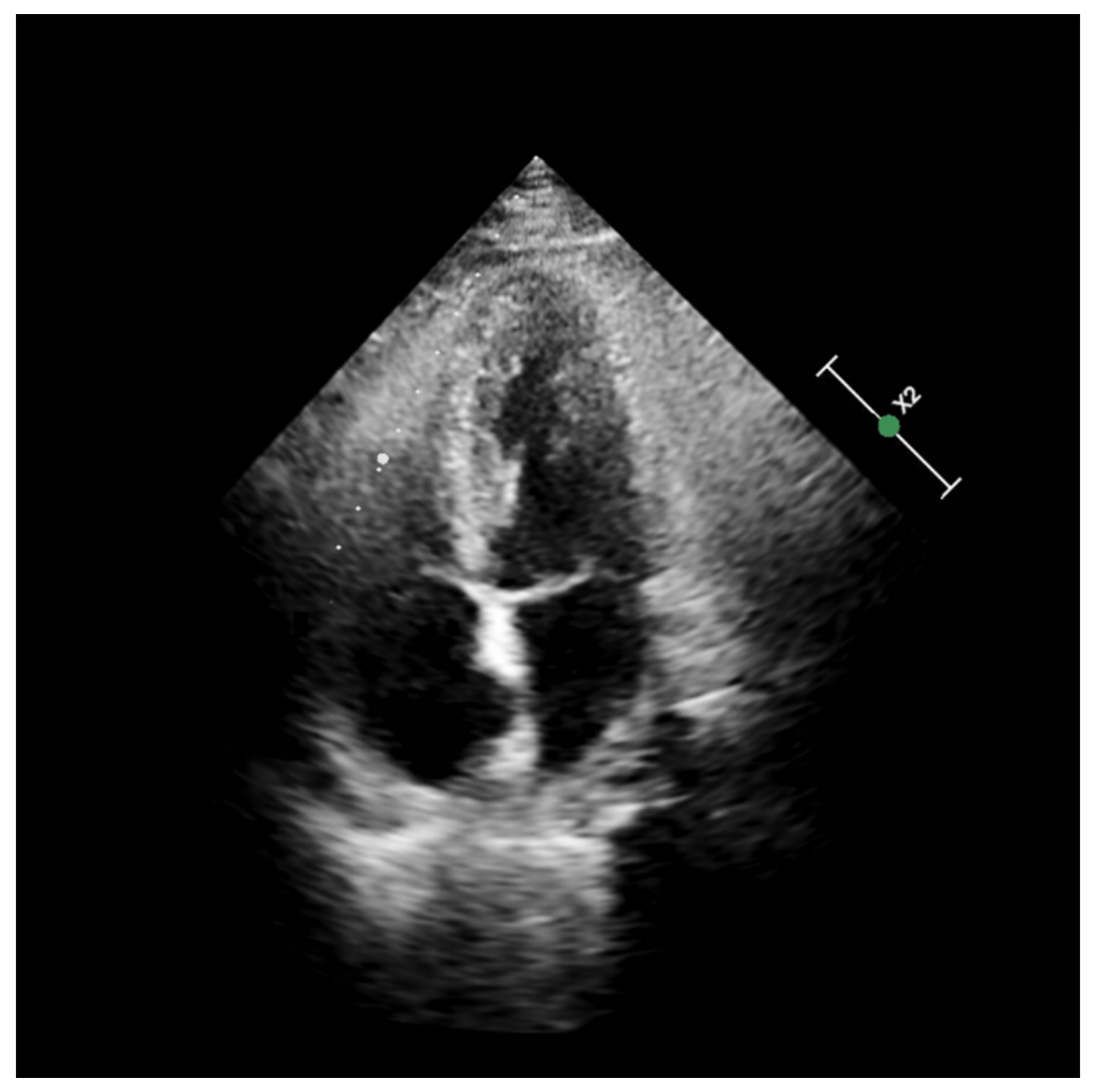

Figure 4: Transthoracic echocardiogram in apical four-chamber view showing no residual cardiac masses after surgery.

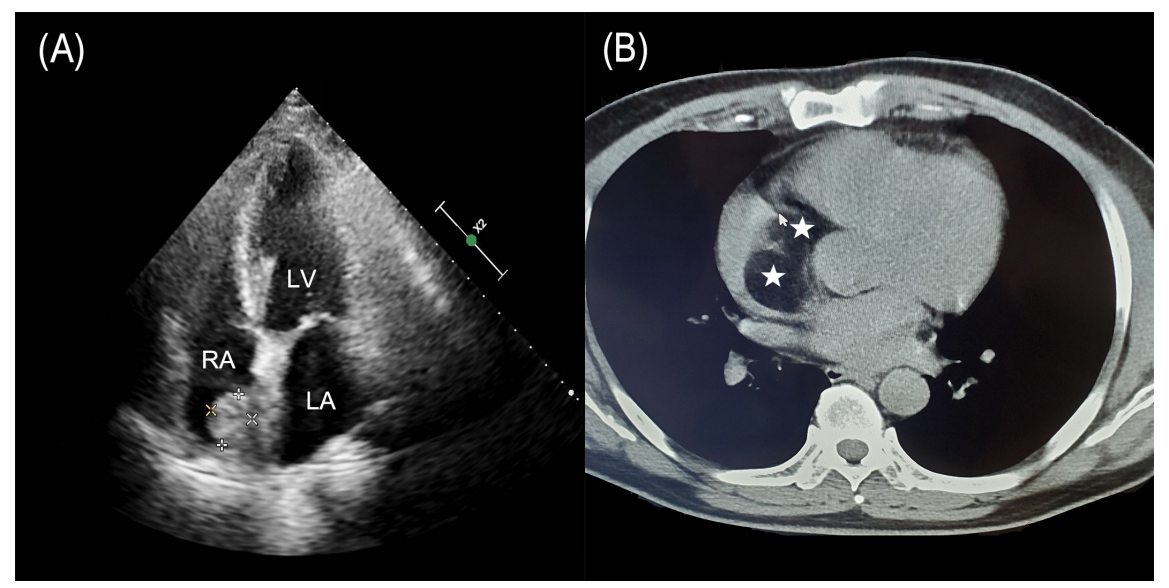



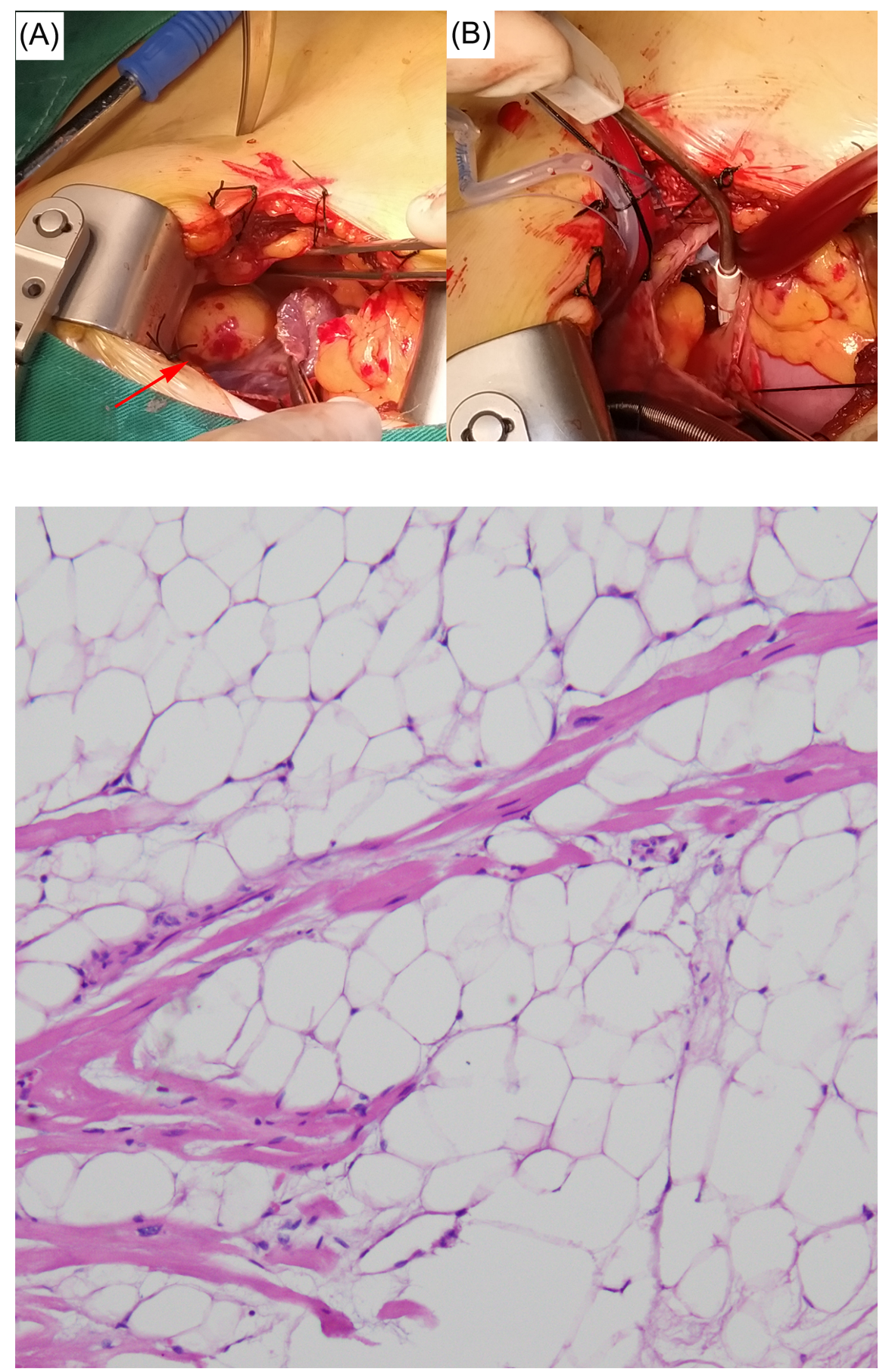


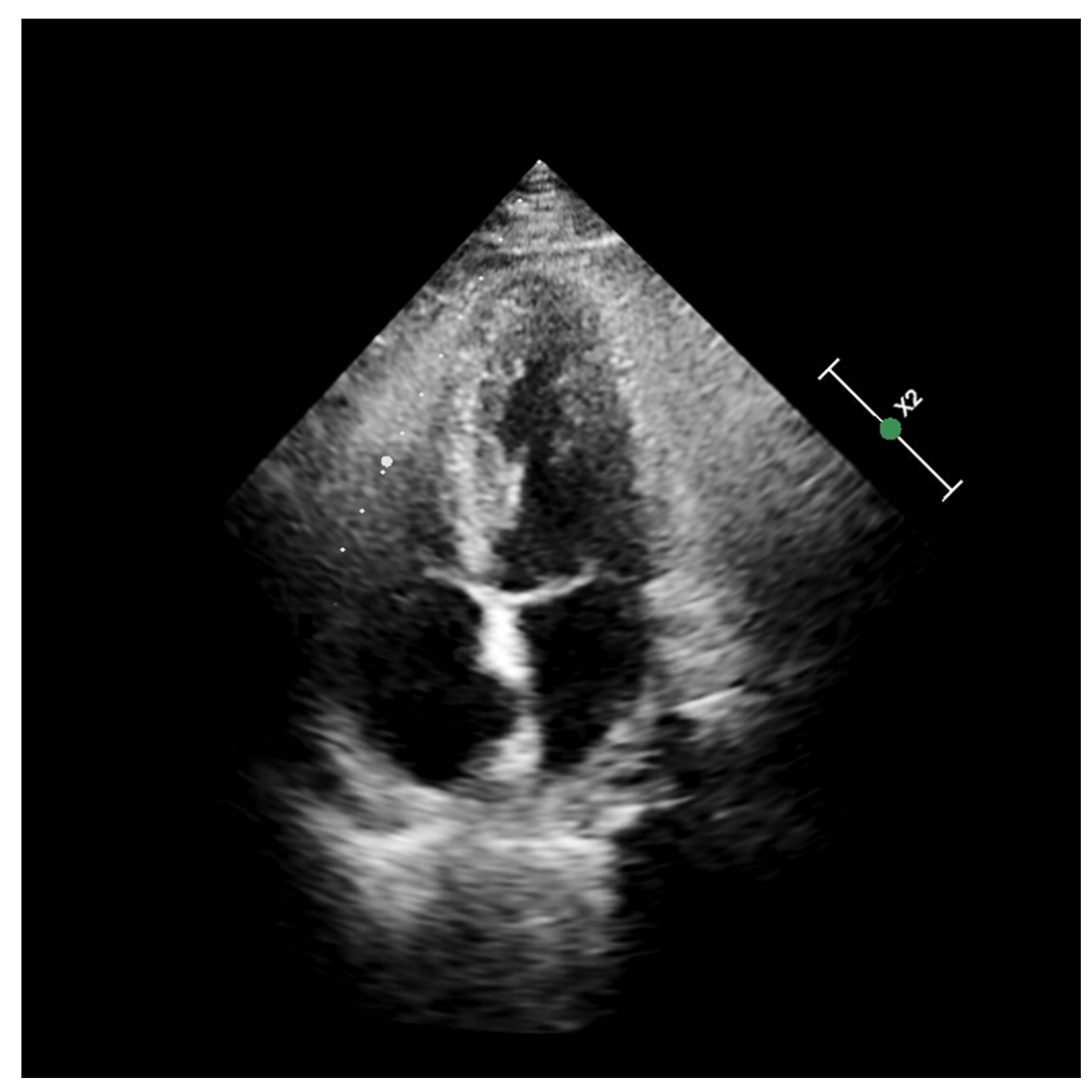

